compared by logistic regression. $\mathrm{P}$ values $<0.05$ were taken as statistically significant.

Results Amongst 929552 patients admitted during the study period, 80172 had a diagnosis of psychiatric disease. Mean age of psychiatric patients was 53.9 years, 50.8\% were male and $84.0 \%$ were Caucasian. 22679 (28.3\%) psychiatric patients died. Logistic regression showed mortality was increased significantly by comorbid diagnoses of ischaemic heart disease (OR 1.221), atrial fibrillation (OR 1.357), cerebrovascular disease (OR 1.657), heart failure (OR 2.555), ischaemic stroke (OR 1.386), myocardial infarction (OR 1.234), peripheral vascular disease (OR 1.581), type 1 diabetes (OR 1.836) and type 2 diabetes (OR 1.171). Conversely mortality was significantly reduced in psychiatric patients with comorbid hypertension (OR 0.630), hyperlipidaemia (OR 0.416) and angina (OR 0.779).

Conclusion We have demonstrated that cardiovascular comorbidities are highly prevalent amongst psychiatric patients and contribute significantly to mortality. We also demonstrate that diagnoses of some cardiovascular risk factors (hypertension and hyperlipidaemia, but not diabetes) has a protective effect on mortality, probably due to effective monitoring and management of risk factors. Improved management of cardiovascular risk amongst psychiatric patients could prevent mortality in this at-risk group.

\section{IMPACT OF ETHNICITY ON MORTALITY AMONGST TOBACCO ABUSERS IN THE UNITED KINGDOM}

${ }^{1}$ Paul R Carter*, 'Jennifer Reynolds, ${ }^{2}$ Andrew Carter, ${ }^{2}$ Hardeep Uppal, ${ }^{3}$ Suresh Chandran, ${ }^{4}$ Jaydeep Sarma, ${ }^{2}$ Rahul Potluri. ${ }^{1}$ Royal Free London NHS Foundation Trust; ${ }^{2}$ ACALM Study Unit in Collaboration with Aston Medical School; ${ }^{3}$ Department of Acute Medicine, North Western Deanery; ${ }^{4}$ University Hospital South Manchester; * Presenting Author

\subsection{6/heartjnl-2016-309890.78}

Introduction Tobacco smoking contributes significantly to the global health burden and its negative impact on cardiovascular morbidity and mortality in particular have been well documented. Smoking is affected by sociodemographic factors though with rates varying according to sex, social class and ethnicity. However, the impact of ethnicity on mortality amongst hospitalised tobacco abusers is yet to be studied.

Objectives We aimed to investigate the impact of ethnicity on mortality of hospitalised patients with a comorbid diagnosis of tobacco abuse. We did this using a large database of patients admitted with comorbid tobacco abuse to hospitals in the North of England between 2000-2013.

Methods Anonymous information on adult tobacco abusers was obtained for hospitals in North West England between $1^{\text {st }}$ January 2000 and $31^{\text {st }}$ March 2013. This data was analysed according to the ACALM (Algorithm for Comorbidities, Associations, Length of stay and Mortality) study protocol. ICD-10 and OPCS-4 codes were used to trace patients coded for tobacco abuse, patient demographics and mortality data.

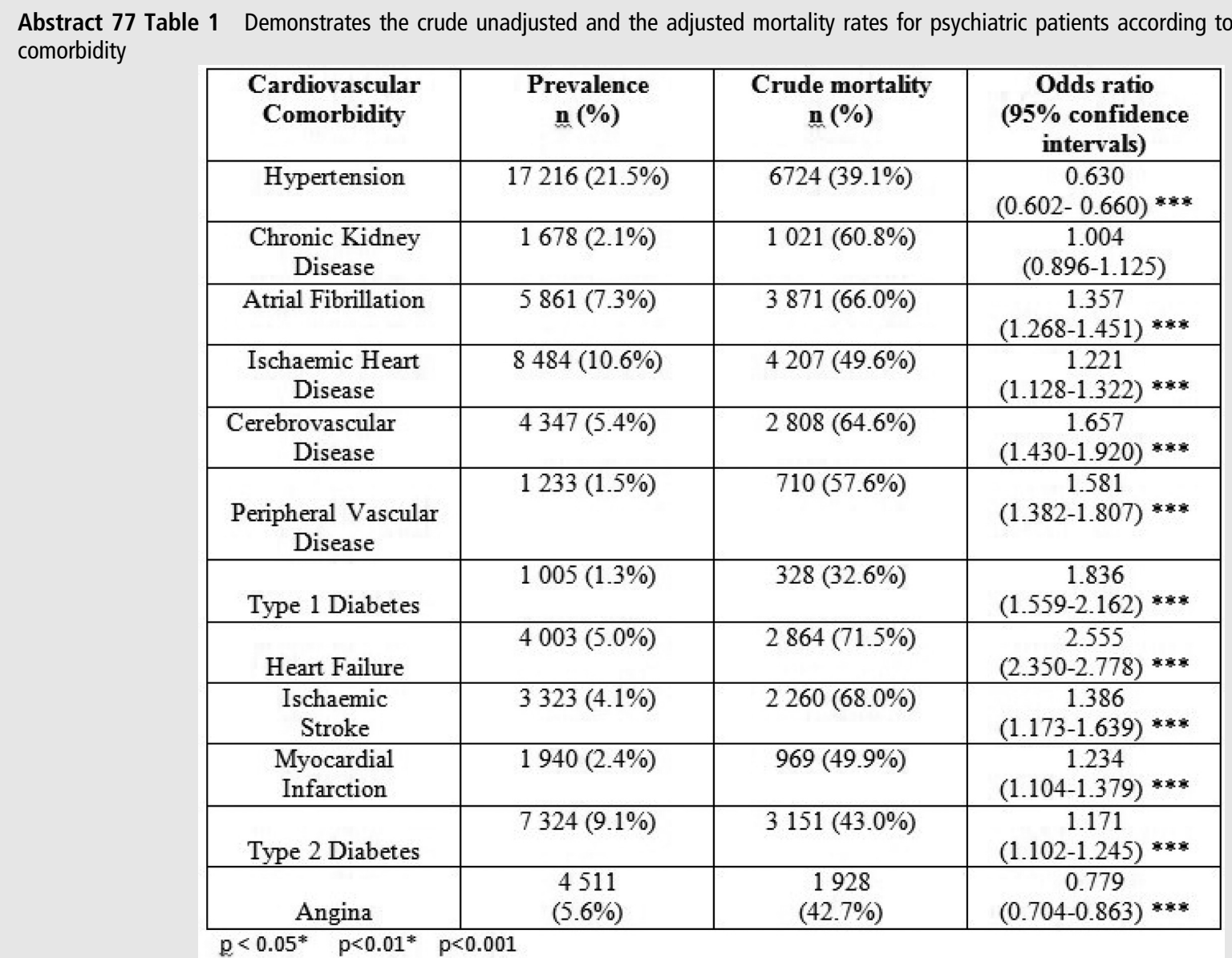


Mortality of tobacco abuse patients was compared by logistic regression. $\mathrm{P}$ values of $<0.05$ were taken as statistically significant.

Results 28379 patients had comorbid tobacco abuse during the study period. Mean age of tobacco abusers was 44.9 years, $51.2 \%$ were male and $1918(6.8 \%)$ tobacco abuse patients died. The majority were Caucasian $(84.8 \%)$ with lower proportions of South Asian (4.7\%), Afro-Caribbean (1.9\%), Oriental $(0.2 \%)$, mixed (1.0\%), other $(2.5 \%)$ and unknown $(4.8 \%)$ ethnicities. Crude mortality was highest amongst Caucasian patients $(7.3 \%)$ compared to South Asian (2.2\%), AfroCaribbean (2.7\%), Oriental (1.7\%), mixed (0.3\%), other (3.1\%) and patients with unknown ethnicities (6.1\%). Of all these ethnic groups, logistic regression accounting for age and sex showed that only South Asian ethnic origin affected mortality rates amongst patients with comorbid tobacco abuse. South Asian patients were 1.90 times more likely to die $\mathbf{9 5 \%}$ CI 1.21-2.97).

Conclusion Our results demonstrate that mortality of tobacco abusers varies according to ethnicity with higher mortality amongst South Asian patients in particular. This could represent differences in smoking rates or an increased susceptibility to smoking. This is particularly important given that smoking and South Asian ethnicity are both known risk factors for developing cardiovascular disease.

\section{HYPERLIPIDAEMIA REDUCES MORTALITY IN BREAST, PROSTATE, LUNG AND BOWEL CANCER}

${ }^{1}$ Paul R Carter*, ${ }^{2}$ John Mcgowan, ${ }^{3}$ Hardeep Uppal, ${ }^{4}$ Suresh Chandran, ${ }^{5}$ Jaydeep Sarma, ${ }^{3}$ Rahul Potluri. ${ }^{1}$ The Royal Free London NHS Foundation Trust; ${ }^{2}$ Central Manchester University Hospitals NHS Foundation Trust; ${ }^{3}$ ACALM Study Unit in Collaboration with Aston Medical School; ${ }^{4}$ Department of Acute Medicine, North Western Deanery; ${ }^{5}$ University Hospital South Manchester, *Presenting Author

10.1136/heartjnl-2016-309890.79
Introduction Hyperlipidaemia is a well -established cardiovascular risk factor but the effect of hyperlipidaemia and treatment with cholesterol-lowering drugs on cancer remain equivocal. We aimed to investigate the impact of comorbid diagnosis of hyperlipidaemia amongst patients with the four most prevalent cancer types in the United Kingdom (Lung, Breast, Prostate and Bowel). We did this using a large database of patients admitted with comorbid hyperlipidaemia to hospitals in the North of England, UK between 2000-2013.

Methods Anonymous information on patients with a primary diagnosis of lung, breast, prostate and bowel cancers were obtained from hospitals in North England, UK between $1^{\text {st }}$ January 2000 and $31^{\text {st }}$ March 2013. This data was analysed according to the ACALM (Algorithm for Comorbidities, Associations, Length of stay and Mortality) study protocol. ICD-10 and OPCS-4 codes were used to trace patients coded for cancer, patient demographics, prevalence of hyperlipidaemia and mortality data. The impact of hyperlipidaemia on mortality in cancer patients was analysed by cox regression adjusted for age, gender and ethnicity. $\mathrm{P}$ values of $<0.05$ were taken as statistically significant.

Results 929552 patients were admitted during the study period. Of these 7997 had lung cancer, 5481 had breast cancer, 4629 had prostate cancer, and 4570 had bowel cancer. Comorbid diagnoses of hyperlipidaemia significantly reduced mortality amongst patients with all four cancer types studied. Cox regression analyses accounting for differences in age, gender and ethnicity showed that hyperlipidaemia was associated with a significantly reduced mortality rate in lung cancer (OR 0.78, 95\% CI 0.70-0.87), breast cancer (OR 0.57, 95\% CI $0.43-0.77$ ), prostate cancer (OR $0.53,95 \%$ CI $0.50-0.79$ ) and bowel cancer (OR 0.70, 95\% CI 0.58-0.84).

Conclusion We have demonstrated for the first time that comorbid hyperlipidaemia has a highly protective effect on mortality amongst patients with the four most prevalent cancers in the UK. The underlying reasons for this are yet to be determined but treatment with statins may contribute. This

Abstract 78 Table 1 Demonstrates the crude unadjusted and the adjusted mortality rates for tobacco users according to ethnicity

\begin{tabular}{|c|c|c|c|}
\hline Ethnicity & $\begin{array}{c}\text { Prevalence } \\
\mathbf{n}(\%)\end{array}$ & $\begin{array}{c}\text { Crude mortality } \\
\mathbf{n}(\%)\end{array}$ & $\begin{array}{c}\text { Odds ratio } \\
\text { (95\% confidence } \\
\text { intervals) }\end{array}$ \\
\hline Caucasian & $24077(84.8 \%)$ & $1767(7.3 \%)$ & $0.80(\mathrm{Cl} 0.62-1.01)$ \\
\hline South Asian & $1334(4.7 \%)$ & $29(2.2 \%)$ & $1.90(\mathrm{Cl} 1.21-2.97){ }^{*}$ \\
\hline Afro-Caribbean & $546(1.9 \%)$ & $15(2.7 \%)$ & $1.48(\mathrm{Cl} 0.83-2.65)$ \\
\hline Oriental & $59(0.2 \%)$ & $1(1.7 \%)$ & $3.54(\mathrm{Cl} 0.47-26.99)$ \\
\hline Mixed & $293(1.0 \%)$ & $1(0.3 \%)$ & $5.85(\mathrm{Cl} 0.80-42.63)$ \\
\hline Other & $719(2.5 \%)$ & $22(3.1 \%)$ & $1.25(\mathrm{Cl} 0.75-2.06)$ \\
\hline Unknown & $1351(4.8 \%)$ & $83(6.1 \%)$ & \\
\hline
\end{tabular}

$\mathrm{p}<0.05^{*}$ 